\title{
New Results on the Performance Evaluation of the Relay Fading Channel
}

\author{
Theodoros A. Tsiftsis ${ }^{* \dagger}$, George K. Karagiannidis ${ }^{\ddagger}$ and P. Takis Mathiopoulos* \\ *Institute for Space Applications \& Remote Sensing, National Observatory of Athens, \\ Email:mathio@space.noa.gr \\ $\dagger^{\dagger}$ Department of Electrical \& Computer Engineering, University of Patras, \\ Email:tsiftsis@ee.upatras.gr \\ ${ }^{\dagger}$ Department of Electrical \& Computer Engineering, Aristotle University of Thessaloniki, \\ Email: geokarag@auth.gr
}

\begin{abstract}
The end-to-end performance of a dual-hop relaying system operating over independent, non-identical Nakagami$m$ fading channels, is analyzed and evaluated. Closed-form expressions for the cumulative distribution function, the probability density function, the moments and the moment generating function of the end-to-end signal-to-noise ratio (SNR), are derived. Using these results, closed-form expressions for the outage probability are presented for both channel state information and fixed-gain relays. Furthermore, for the case of fixed-gain relay, the average end-to-end SNR, the amount of fading and the average bit-error rate are also expressed in closed-form. The proposed mathematical analysis is complemented by numerical examples, including the effects on the overall performance of the SNRs unbalancing as well as the fading severity.
\end{abstract}

\section{INTRODUCTION}

Relaying technology is a promising solution for the throughput and high data rates coverage requirements in future cellular and ad-hoc wireless communications systems. There are two main advantages of this relaying technology:

1) Very low transmit RF power requirements;

2) Use of multiuser spatial diversity to combat fading.

Recently, the concept of cooperative diversity, where the mobile users cooperate/collaborate with each other in order to exploit the benefits of spatial diversity without the need of employing antenna arrays, has gained great interest [1]-[7]. More specifically, Emamian et al. in [1], studied the multiuser spatial diversity systems with channel state information (CSI)-based relays, as first proposed in [5] and later in [6], and derived closed-form expressions for the outage probability in Rayleigh fading. In [2], Anghel and Kaveh have presented tight bounds for the outage and error probability for a distributed spatial diversity wireless system, also in Rayleigh fading. In another contribution, Hasna and Alouini proposed bounds for the outage and the error performance of dual-hop systems with regenerative and non-regenerative CSI-based relays over Rayleigh [8], and Nakagami- $m$ fading channels [9]. Note, that the relay considered in [2], [8], [9] is an ideal type of CSI-based relay, where the noise figure has been ignored from the relay gain. In another contribution, Sendonaris et al. in [3], [4] proposed the user cooperation concept and considered practical issues related to its implementation.

In this paper we present for the first time a completely analytical approach in obtaining an end-to-end performance evaluation of the relay Nakagami- $m$ fading channels. In doing so, we first derive closed-form expressions for the statistics [i.e., probability density function (PDF), cumulative distribution function (CDF), moments and moment generating function (MGF)] of the end-to-end signal-to-noise ratio (SNR). These new results are then applied to study the end-to-end performance of dual-hop wireless communication systems over Nakagami- $m$ fading channels such as average end-toend SNR, amount of fading $(\mathrm{AOF})$ and average bit-error rate (BER). Furthermore, in order to verify our mathematical analysis, it will be shown that our general expressions for Nakagami- $m$ fading reduce to previously published results for $m=1$ (i.e., Rayleigh fading).

\section{System And Channel Model}

We consider a dual-hop relaying system operating over independent and non-identical Nakagami- $m$ fading channels. The source-terminal S communicates with the destination-terminal $\mathrm{D}$ through terminal $\mathrm{R}$ which acts as a non-regenerative gain relay. Assuming that $S$ is transmitting a signal with an average power normalized to unity, then, the instantaneous equivalent end-to-end SNR of the dual-hop path, can be expressed as [8]-[10]

$$
\gamma_{e n d}=\frac{\left(\alpha_{1}^{2} / N_{0,1}\right)\left(\alpha_{2}^{2} / N_{0,2}\right)}{\left(\alpha_{2}^{2} / N_{0,2}\right)+\left(1 / g^{2} N_{0,1}\right)}
$$

where $\alpha_{i}$ is the fading amplitude of the $i$ th hop $(i=1,2)$ $N_{0, i}$ is the one sided power spectral density at the input of $\mathrm{R}$ and $\mathrm{D}$ respectively, and $g$ is the gain of the relay. Since, $\alpha_{i}$ is modeled as a Nakagami- $m$ random variable (RV), the instantaneous SNR, $\gamma_{i}=\alpha_{i}^{2} / N_{0}$, is a Gamma distributed RV with following probability density function (PDF)

$$
f_{\gamma_{i}}(\gamma)=\frac{m_{i}^{m_{i}}}{\bar{\gamma}_{i}^{m_{i}} \Gamma\left(m_{i}\right)} \gamma^{m_{i}-1} \exp \left(-\frac{m_{i} \gamma}{\bar{\gamma}_{i}}\right)
$$

where $\Gamma(\cdot)$ is the Gamma function $[11$, eq. $(8.310 / 1)], m_{i} \geq$ $1 / 2$ is a parameter describing the fading severity and $\bar{\gamma}_{i}$ the average SNR of the $i$ th hop. When R has available CSI from the first hop and its gain aims to limit the output power of the relay, one kind of gain proposed by Laneman and Wornell in [5] is given by 


$$
g_{1}^{2}=\frac{1}{\alpha_{1}^{2}+N_{0,1}} .
$$

Therefore, the instantaneous equivalent end-to-end SNR of the dual-hop path, can be expressed as [1], [2], [8], [9]

$$
\gamma_{e q_{1}}=\frac{\gamma_{1} \gamma_{2}}{\gamma_{1}+\gamma_{2}+1}
$$
by

When $\mathrm{R}$ introduces fixed-gain to the received signal given

$$
g_{2}^{2}=\frac{1}{C N_{0,1}}
$$

where $C$ is a positive constant, the instantaneous end-to-end SNR of the dual-hop path, can be written as [12]

$$
\gamma_{e q_{2}}=\frac{\gamma_{1} \gamma_{2}}{C+\gamma_{2}}
$$

\section{Performance Analysis of the Dual-HoP RELAY FADING CHANNEL}

\section{A. CSI-based Gain Relay}

1) Outage Probability : The end-to-end outage probability at $\mathrm{D}$ is defined as the probability that the equivalent output SNR, $\gamma_{e q 1}$, falls bellow a given threshold, $\gamma_{t h}$. Consequently, outage probability is given by

$$
P_{\text {out }}=\operatorname{Pr}\left[\gamma_{e q 1} \leq \gamma_{t h}\right]=F_{\gamma_{e q 1}}\left(\gamma_{t h}\right)
$$

where $F_{\gamma_{e q 1}}\left(\gamma_{t h}\right)$ is the CDF of the instantaneous end-toend SNR for the CSI-based dual-hop relay fading channel, evaluated at $\gamma=\gamma_{t h}$. Using (4), $F_{\gamma_{e_{1}}}(\gamma)$ can be expressed as

$$
\begin{aligned}
F_{\gamma_{\text {eq }}}(\gamma) & =\int_{0}^{\infty} \operatorname{Pr}\left[\frac{\gamma_{1} \gamma_{2}}{\gamma_{1}+\gamma_{2}+1} \leq \gamma \mid \gamma_{2}\right] f_{\gamma_{2}}\left(\gamma_{2}\right) d \gamma_{2} \\
& =\int_{0}^{\gamma} \operatorname{Pr}\left[\gamma_{1} \geq \frac{\gamma\left(\gamma_{2}+1\right)}{\gamma_{2}-\gamma} \mid \gamma_{2}\right] f_{\gamma_{2}}\left(\gamma_{2}\right) d \gamma_{2} \\
& +\int_{\gamma}^{\infty} \operatorname{Pr}\left[\gamma_{1} \leq \frac{\gamma\left(\gamma_{2}+1\right)}{\gamma_{2}-\gamma} \mid \gamma_{2}\right] f_{\gamma_{2}}\left(\gamma_{2}\right) d \gamma_{2} \\
& =\mathrm{I}_{1}+\mathrm{I}_{2}
\end{aligned}
$$

where

$$
\mathbf{I}_{1}=\int_{0}^{\gamma} f_{\gamma_{2}}\left(\gamma_{2}\right) d \gamma_{2}=F_{\gamma_{2}}(\gamma)=1-\frac{\Gamma\left(m_{2}, \frac{m_{2}}{\bar{\gamma}_{2}} \gamma\right)}{\Gamma\left(m_{2}\right)}
$$

and $\Gamma(\cdot, \cdot)$ is the incomplete Gamma function defined in $[11$, eq. $(8.350 .2)]$,

$$
\begin{aligned}
\mathrm{I}_{2} & =\int_{\gamma}^{\infty} F_{\gamma_{1}}\left[\gamma\left(\frac{\gamma_{2}+1}{\gamma_{2}-\gamma}\right)\right] f_{\gamma_{2}}\left(\gamma_{2}\right) d \gamma_{2} \\
& =\int_{\gamma}^{\infty}\left\{1-\frac{\Gamma\left[m_{1}, \frac{m_{1} \gamma}{\bar{\gamma}_{1}}\left(\frac{\gamma_{2}+1}{\gamma_{2}-\gamma}\right)\right]}{\Gamma\left(m_{1}\right)}\right\} f_{\gamma_{2}}\left(\gamma_{2}\right) d \gamma_{2} \\
& =\frac{\Gamma\left(m_{2}, \frac{m_{2} \gamma}{\bar{\gamma}_{2}}\right)}{\Gamma\left(m_{2}\right)}-\frac{m_{2}^{m_{2}}}{\bar{\gamma}_{2}^{m_{2}} \Gamma\left(m_{1}\right) \Gamma\left(m_{2}\right)} \mathrm{I}_{3}
\end{aligned}
$$

and

$$
\begin{aligned}
\mathrm{I}_{3}=\int_{\gamma}^{\infty} \gamma_{2}^{m_{2}-1} & \exp \left(-\frac{m_{2} \gamma_{2}}{\bar{\gamma}_{2}}\right) \\
& \times \Gamma\left[m_{1}, \frac{m_{1} \gamma}{\bar{\gamma}_{1}}\left(\frac{\gamma_{2}+1}{\gamma_{2}-\gamma}\right)\right] d \gamma_{2}
\end{aligned}
$$

Since the integral $I_{3}$ is not a tabulated one a closed form solution is proposed in Appendix. From (8) and by using (A-4), (9) and (10), after simple algebraic manipulations $F_{\gamma_{e q_{1}}}(\gamma)$ can be expressed as

$$
\begin{aligned}
F_{\gamma_{e q 1}}(\gamma) & =1-\frac{2 m_{2}^{m_{2}}\left(m_{1}-1\right) ! \exp \left[-\left(\frac{m_{1} \gamma}{\bar{\gamma}_{1}}+\frac{m_{2} \gamma}{\bar{\gamma}_{2}}\right)\right]}{\bar{\gamma}_{2}^{m_{2}} \Gamma\left(m_{1}\right) \Gamma\left(m_{2}\right)} \\
& \times \sum_{k=0}^{m_{1}-1} \sum_{\ell=0}^{k} \sum_{r=0}^{m_{2}-1}\left\{\frac{1}{k !}\left(\begin{array}{l}
k \\
l
\end{array}\right)\left(\begin{array}{c}
m_{2}-1 \\
r
\end{array}\right)\right. \\
& \times\left(\frac{m_{2}}{\bar{\gamma}_{2}}\right)^{\frac{\ell-r-1}{2}}\left(\frac{m_{1}}{\bar{\gamma}_{1}}\right)^{\frac{2 k-\ell+r+1}{2}} \gamma^{\frac{2 k+2 m_{2}-\ell-r-1}{2}} \\
& \left.\times(\gamma+1)^{\frac{\ell+r+1}{2}} K_{\ell-r-1}\left[2 \sqrt{\frac{m_{1} m_{2} \gamma(1+\gamma)}{\bar{\gamma}_{1} \bar{\gamma}_{2}}}\right]\right\} .
\end{aligned}
$$

where (.) denotes the binomial coefficient and $K_{\nu}[\cdot]$ is the $\nu$ th order modified Bessel function of the second kind, defined in [11, eq. (8.432)].

Note, that for the case of $m_{i}=1$, i.e., Rayleigh fading channels, (12) reduces to the previously published result [1, eq. (14)].

\section{B. Fixed-Gain Relay}

1) Outage Probability: The CDF of the instantaneous endto-end SNR for the fixed-gain dual-hop relay fading channel, $F_{\gamma_{\text {eq2 }}}(\gamma)$, using $(6)$, can be written as

$$
\begin{aligned}
F_{\gamma_{e q}}(\gamma) & =\int_{0}^{\infty} \operatorname{Pr}\left[\frac{\gamma_{1} \gamma_{2}}{\gamma_{2}+C} \leq \gamma \mid \gamma_{2}\right] f_{\gamma_{2}}\left(\gamma_{2}\right) d \gamma_{2} \\
& =\int_{0}^{\infty} \operatorname{Pr}\left[\gamma_{1} \leq \frac{\gamma\left(\gamma_{2}+C\right)}{\gamma_{2}} \mid \gamma_{2}\right] f_{\gamma_{2}}\left(\gamma_{2}\right) d \gamma_{2} \\
& =\int_{0}^{\infty}\left\{1-\frac{\Gamma\left[m_{1}, \frac{m_{1} \gamma}{\gamma_{1}}\left(\frac{\gamma_{2}+C}{\gamma_{2}}\right)\right]}{\Gamma\left(m_{1}\right)}\right\} f_{\gamma_{2}}\left(\gamma_{2}\right) d \gamma_{2} \\
& =1-\frac{m_{2}^{m_{2}}}{\bar{\gamma}_{2}^{m_{2}} \Gamma\left(m_{1}\right) \Gamma\left(m_{2}\right)} \mathcal{I}_{1}
\end{aligned}
$$

with

$$
\begin{aligned}
\mathcal{I}_{1}=\int_{0}^{\infty} & \gamma_{2}^{m_{2}-1} \exp \left(-\frac{m_{2} \gamma_{2}}{\bar{\gamma}_{2}}\right) \\
& \times \Gamma\left[m_{1}, \frac{m_{1} \gamma}{\bar{\gamma}_{1}}\left(\frac{\gamma_{2}+C}{\gamma_{2}}\right)\right] d \gamma_{2}
\end{aligned}
$$

Since the integral $\mathcal{I}_{1}$ is not a tabulated one, it can be solved 
in closed-form as (see Appendix)

$$
\begin{aligned}
\mathcal{I}_{1} & =\sum_{k=0}^{m_{1}-1} \sum_{\ell=0}^{k} \frac{2\left(m_{1}-1\right) !}{k !} \exp \left(-\frac{m_{1} \gamma}{\bar{\gamma}_{1}}\right)\left(\begin{array}{l}
k \\
\ell
\end{array}\right) \\
& \times C^{k-\ell}\left(\frac{m_{1} \gamma}{\bar{\gamma}_{1}}\right)^{k}\left(\frac{m_{2}}{\bar{\gamma}_{2}}\right)^{\frac{k-\ell-m_{2}}{2}}\left(\frac{\bar{\gamma}_{1}}{C m_{1} \gamma}\right)^{\frac{k-\ell-m_{2}}{2}} \\
& \times K_{k-m_{2}-\ell}\left(2 \sqrt{\frac{m_{1} m_{2} C \gamma}{\bar{\gamma}_{1} \bar{\gamma}_{2}}}\right) .
\end{aligned}
$$

Using (13) and (15), $F_{\gamma_{\text {eq }}}(\gamma)$ can be written as

$$
\begin{aligned}
F_{\gamma_{\ell_{2}}}(\gamma) & =1-\sum_{k=0}^{m_{1}-1} \sum_{\ell=0}^{k} \Xi[k, \ell] \exp \left(-\frac{m_{1} \gamma}{\bar{\gamma}_{1}}\right) \\
& \times \gamma^{\frac{k+\ell+m_{2}}{2}} K_{k-m_{2}-\ell}(2 \sqrt{\tau \gamma})
\end{aligned}
$$

where

$$
\begin{aligned}
\Xi[k, \ell]= & 2\left(\begin{array}{c}
k \\
\ell
\end{array}\right) \frac{\left(m_{1}-1\right) ! C^{\left(k+m_{2}-\ell\right) / 2}}{\Gamma\left(m_{1}\right) \Gamma\left(m_{2}\right) k !} \\
& \times\left(\frac{m_{1}}{\bar{\gamma}_{1}}\right)^{\left(k+m_{2}+\ell\right) / 2}\left(\frac{m_{2}}{\bar{\gamma}_{2}}\right)^{\left(k+m_{2}-\ell\right) / 2}
\end{aligned}
$$

and

$$
\tau=\frac{m_{1} m_{2} C}{\bar{\gamma}_{1} \bar{\gamma}_{2}}
$$

It is obvious from (16) that $P_{\text {out }}$ for dual-hop systems with fixed-gain relays over Nakagami- $m$ fading channels equals to $F_{\gamma_{e q_{2}}}\left(\gamma_{t h}\right)$.

Note, that for the case of $m_{i}=1,(16)$ reduces to a previously published result [12, eq. (9)].

2) Moments: The $n$th order moment of the end-to-end SNR at terminal $\mathrm{D}$ is given by

$$
\mu_{n}=\mathcal{E}\left\langle\gamma_{e q_{2}}^{n}\right\rangle=\int_{0}^{\infty} \gamma^{n} f_{\gamma_{e q_{2}}}(\gamma) d \gamma
$$

where $\mathcal{E}\langle\cdot\rangle$ denotes the statistical average operator. Taking the derivative of (16) with respect to $\gamma$, one can obtain

$$
\begin{aligned}
& f_{\gamma_{\text {eq }}}(\gamma)=\sum_{k=0}^{m_{1}-1} \sum_{\ell=0}^{k} \Xi[k, \ell] \exp \left(-\frac{m_{1} \gamma}{\bar{\gamma}_{1}}\right) \\
& \times\left[\frac{m_{1}}{\bar{\gamma}_{1}} \gamma^{\left(k+\ell+m_{2}\right) / 2} K_{k-\ell-m_{2}}(2 \sqrt{\tau \gamma})\right. \\
& \quad+\frac{\sqrt{\tau}}{2} \gamma^{\left(k+\ell+m_{2}-1\right) / 2} K_{k-\ell-m_{2}-1}(2 \sqrt{\tau \gamma}) \\
& \quad+\frac{\sqrt{\tau}}{2} \gamma^{\left(k+\ell+m_{2}-1\right) / 2} K_{k+1-\ell-m_{2}}(2 \sqrt{\tau \gamma}) \\
& \left.\quad-\frac{1}{2}\left(k+\ell+m_{2}\right) \gamma^{\left(k+\ell+m_{2}-2\right) / 2} K_{k-\ell-m_{2}}(2 \sqrt{\tau \gamma})\right] .
\end{aligned}
$$

By substituting (19) into (18), the resulting integral can be solved using [11, eq. (6.643.3)] yielding in a complicated closed-form mathematical expression involving the Whittaker function. This expression can be simplified by applying the identities [11, eqs. (9.220.3) and (9.220.4)] of the well-known confluent hypergeometric function ${ }_{1} F_{1}(x, y, ; z)$ defined in [11, eqs. (9.210.1) and (9.220.4)], as follows:

$$
\left.\left.\begin{array}{c}
\mu_{n}=\sum_{k=0}^{m_{1}-1} \sum_{\ell=0}^{k} \Xi[k, \ell] \\
\times\left\{\xi _ { 1 } [ k , \ell , n ] \left[\frac{\left[{ }_{1} F_{1}\left(k+n, k-m_{2}-\ell ; \frac{\bar{\gamma}_{1} \tau}{m_{1}}\right)\right.}{\Gamma\left(1-k+m_{2}+\ell\right)^{-1}}\right.\right. \\
\left.-\frac{{ }_{1} F_{1}\left(k+n, 1+k-m_{2}-\ell ; \frac{\bar{\gamma}_{1} \tau}{m_{1}}\right)}{\left(\left(k+m_{2}+\ell\right) \Gamma\left(-k+m_{2}+\ell\right)\right)^{-1}}\right] \\
+\xi_{2}[k, \ell, n]\left[\frac{\left[{ }_{1} F_{1}\left(1+k+n, 1+k-m_{2}-\ell ; \frac{\bar{\gamma}_{1} \tau}{m_{1}}\right)\right.}{\Gamma\left(-k+m_{2}+\ell\right)^{-1}}\right. \\
+\frac{\bar{\gamma}_{1} \tau}{\left.2 m_{1} \frac{{ }_{1} F_{1}\left(1+k+n, 2+k-m_{2}-\ell ; \frac{\bar{\gamma}_{1} \tau}{m_{1}}\right)}{\Gamma\left(-1-k+m_{2}+\ell\right)^{-1}}\right]} \\
+\xi_{3}[k, \ell, n]\left[\frac{{ }_{1} F_{1}\left(m_{2}+n+\ell,-k+m_{2}+\ell ; \frac{\bar{\gamma}_{1} \tau}{m_{1}}\right)}{\Gamma\left(1+k-m_{2}-\ell\right)^{-1}}\right.
\end{array}\right]\right\}
$$

where

$$
\begin{gathered}
\xi_{1}[k, \ell, n]=\frac{1}{2}\left(\frac{m_{1}}{\bar{\gamma}_{1}}\right)^{-k-n} \tau^{\frac{k-m_{2}-\ell}{2}} \Gamma(k+n) \\
\xi_{2}[k, \ell, n]=\left(\frac{m_{1}}{\bar{\gamma}_{1}}\right)^{-k-n} \tau^{\frac{k-m_{2}-\ell}{2}} \Gamma(1+k+n) \\
\xi_{3}[k, \ell, n]=\frac{1}{2}\left(\frac{m_{1}}{\bar{\gamma}_{1}}\right)^{-m_{2}-n-\ell} \tau^{\frac{-k+m_{2}+\ell}{2}} \Gamma\left(m_{2}+n+\ell\right) \\
\xi_{4}[k, \ell, n]=\left(\frac{m_{1}}{\bar{\gamma}_{1}}\right)^{-m_{2}-n-\ell} \tau^{\frac{-k+m_{2}+\ell}{2}} \Gamma\left(1+m_{2}+n+\ell\right) .
\end{gathered}
$$

The above closed-form expression is very useful since it can be used to directly obtain the average end-to-end SNR (i.e., $\mu_{1}$ ) or the AoF since

$$
\mathrm{AoF}=\frac{\mu_{2}}{\mu_{1}^{2}}-1
$$

under various fading channel conditions. 


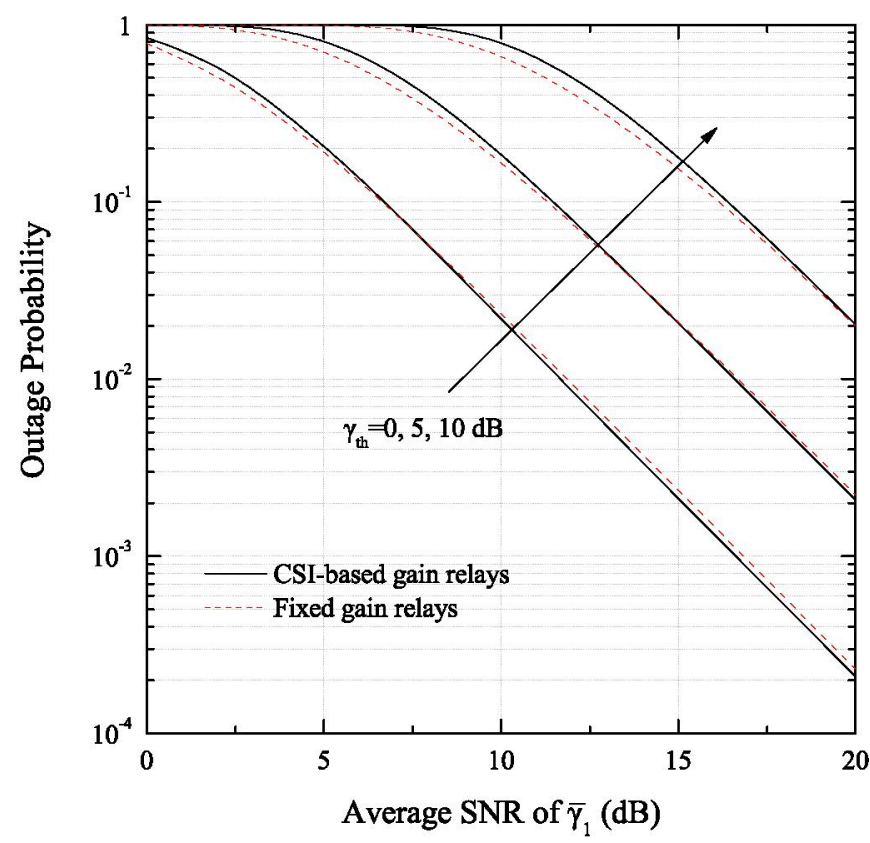

Fig. 1. Outage probability of dual-hop relaying system versus average SNR of first hop, $\bar{\gamma}_{1}$, for different relay types $\left(m=2\right.$ and $\left.\bar{\gamma}_{2}=5 \bar{\gamma}_{1}\right)$.

3) Average BER: The MGF of the end-to-end SNR at terminal $\mathrm{D}$, is defined as [13]

$$
\mathcal{M}_{\gamma_{e q_{2}}}(s) \triangleq \mathcal{E}\left\langle e^{s \gamma_{e q_{2}}}\right\rangle=\int_{0}^{\infty} e^{s \gamma} f_{\gamma_{e q_{2}}}(\gamma) d \gamma
$$

Following the same procedure as for the moments, the MGF for the fixed-gain relay has been derived in closed-form as nested finite sums of confluent hypergeometric functions. Unfortunately, due to space limitations this mathematical expression will not be presented here since it is very complicated and does not provide much insight to the performance analysis. Nevertheless, with the aid of $\mathcal{M}_{\gamma_{\text {eq }}}(s)$, the error performance of a great variety of $M$-ary modulation schemes operating over relay fading channels can be easily obtained [13].

\section{PERFORMANCE RESUlTS AND Discussion}

Using the previously derived expressions, various performance evaluation results have been obtained. Fig. 1 depicts the outage probability as a function of the average SNR of the first hop, for both types of relays and for different outage threshold values. From the comparison between CSI-based relay with an equivalent, in terms of average power consumption fixed-gain relay (i.e, as presented in [12, eq. (13)]), it can be seen that for medium-to-large SNR values the CSI-based relay outperform those with fixed-gain. However, for the low SNR region, dualhop systems with fixed-gain relays slightly outperform those systems with variable gain relays. Considering the higher complexity nature of CSI-based relays, our results show that fixed-gain relays may serve as an efficient solution for relayed transmissions. Similar observations have been made for the Rayleigh fading channel [12]. Furthermore, it is observed that as $\gamma_{t h}$ increases, the SNR range where fixed-gain outperforms

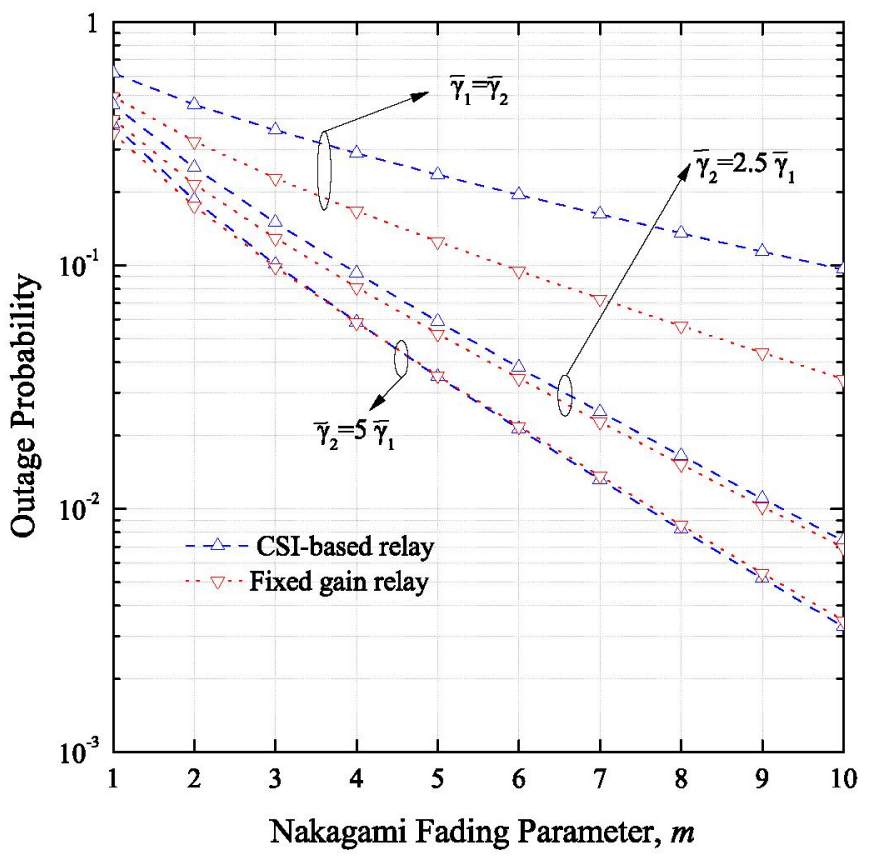

Fig. 2. Outage probability of dual-hop relaying system versus fading parameter $m$, for different relay types $\left(\bar{\gamma}_{1}=\bar{\gamma}_{t h}=5 \mathrm{~dB}\right)$.

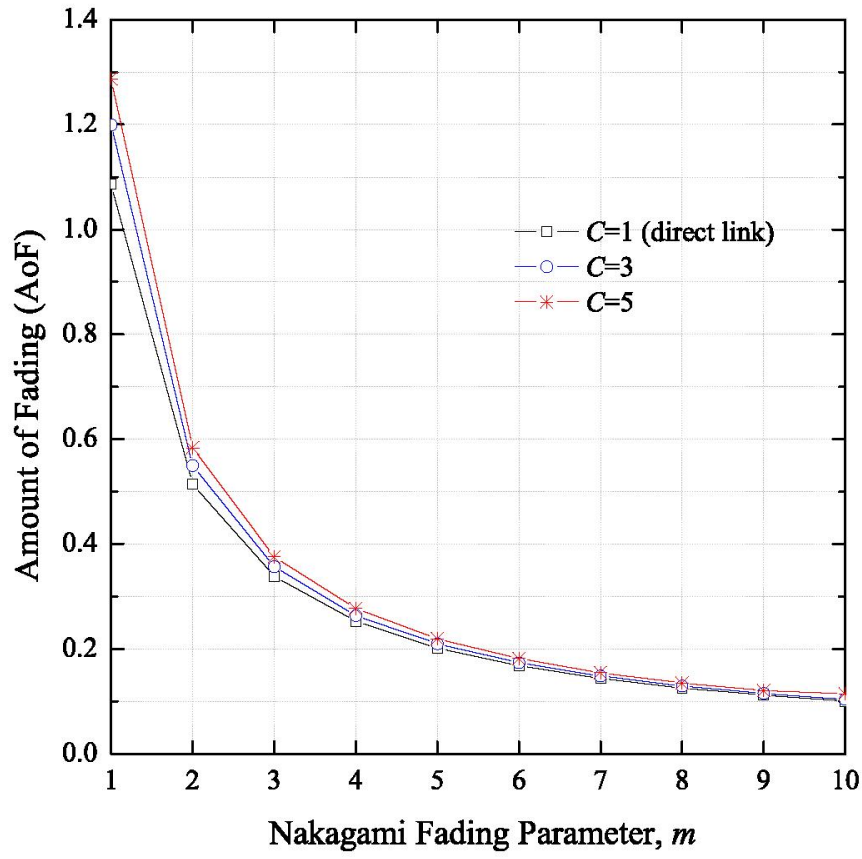

Fig. 3. AoF for fixed-gain relay versus fading severity $\left(m_{1}=m_{2}=m\right.$, $\bar{\gamma}_{2}=25 \mathrm{~dB}$ and $\left.\bar{\gamma}_{1}=5 \mathrm{~dB}\right)$

CSI-based relays also increases. In Fig. 2 similar performance comparisons are presented as function of the fading severity parameter $m$. It is observed that for the same average SNR per hop (i.e., $\bar{\gamma}_{1}=\bar{\gamma}_{2}=\bar{\gamma}$ ), the performance gap between systems with fixed-gain relays and those systems with CSIbased relays is increased, whereas for variable mean hop's 
SNR is decreased. Finally, in Fig. 3 the $\mathrm{AoF}$ of a dual-hop system with fixed-gain relay over integer-order Nakagami $m$ fading parameters, is depicted. In the same figure a comparison with the direct link is presented. It can be seen that $\mathrm{AoF}$ of the relay fading channel is improving with an increase of the parameter $m$ (i.e., less fading). It is interesting to note that AoF deteriorates for low values of $m$ and for high amplification gains. This can be explained by the fact that for strong fading conditions the fixed-gain relay does not amplify only the received signal but also the noise of the first hop.

\section{Conclusions}

In this paper, we presented some new results on the performance of dual-hop communications systems over relay Nakagami- $m$ fading channels. Specifically, closed-form expressions were derived for the outage probability both for CSI-based and fixed-gain relays. Also closed-form expressions were presented for the moments, the $\mathrm{AoF}$ and the the average BER when fixed-gain relay is assumed. Numerical results show that fixed-gain relays may serve as an efficient solution for relayed transmissions regarding the higher complexity nature of CSI-based relays.

\section{APPENDIX \\ EVALUATION OF THE INTEGRAL $\mathbf{I}_{3}$}

The integral $\mathrm{I}_{3}$ in (11), can be written in its general form as

$$
\mathrm{I}_{3}=\int_{y}^{\infty} x^{a-1} \exp (-b x) \Gamma\left[c, d\left(\frac{x+e}{x-y}\right)\right] d x .
$$

where $a, c$, being positive integers and $b, d$, e real numbers. Then, the incomplete Gamma function of the integrand in (A-1), using [11, eq. (8.352.2)] and [11, eq. (1.111)], can be expressed as

$$
\begin{aligned}
\Gamma\left[c, d\left(\frac{x+e}{x-y}\right)\right] & =(c-1) ! \exp (-d) \\
& \times \exp \left[-d\left(\frac{y+e}{x-y}\right)\right] \\
& \times \sum_{k=0}^{c-1} \sum_{\ell=0}^{k} \frac{d^{k}}{k !}\left(\begin{array}{l}
k \\
l
\end{array}\right)\left(\frac{y+e}{x-y}\right)^{\ell} .
\end{aligned}
$$

Using (A-1) and (A-2) after a simple transformation of the integration variable, $\mathrm{I}_{3}$ can be expressed as

$$
\begin{aligned}
& \mathrm{I}_{3}=(c-1) ! \exp [-(d+b y)] \\
& \times \sum_{k=0}^{c-1} \sum_{\ell=0}^{k} \sum_{r=0}^{a-1} \frac{d^{k}}{k !}\left(\begin{array}{c}
k \\
l
\end{array}\right)\left(\begin{array}{c}
a-1 \\
r
\end{array}\right)(y+e)^{\ell} y^{a-r-1} \\
& \quad \times \int_{0}^{\infty} x^{r-\ell} \exp (-b x) \exp \left[-d\left(\frac{y+e}{x}\right)\right] d x .
\end{aligned}
$$

The inner integral of $(A-3)$ can be solved by using [11, eq. (3.471.9)], resulting to

$$
\begin{aligned}
& \mathrm{I}_{3}=(c-1) ! \exp [-(d+b y)] \\
& \times \sum_{k=0}^{c-1} \sum_{\ell=0}^{k} \sum_{r=0}^{a-1} \frac{d^{k}}{k !}\left(\begin{array}{c}
k \\
l
\end{array}\right)\left(\begin{array}{c}
a-1 \\
r
\end{array}\right)(y+e)^{\frac{r+\ell+1}{2}} y^{a-r-1} \\
& \times 2\left(\frac{d}{b}\right)^{\frac{r-\ell+1}{2}} K_{\ell-r-1}[2 \sqrt{b d(y+e)}] .
\end{aligned}
$$

\section{ACKNOWLEDGMENTS}

This work has been performed within the framework of the Satellite Network of Excellence (SatNEx) project, a Network of Excellence (NoE) funded by European Commission (EC) under the FP6 program.

\section{REFERENCES}

[1] V. Emamian, P. Anghel, and M. Kaveh, "Multi-user spatial diversity system in a shadow-fading environment," in Vehicular Technology Conference (Fall VTC'02). Vancouver, BC, Canada: IEEE, 2002, pp. 573-576.

[2] P. A. Anghel and M. Kaveh, "Exact symbol error probability of a cooperative network in a Rayleigh-fading environment," IEEE Trans. Wireless Commun., vol. 3, pp. 1416-1421, Sept. 2004.

[3] A. Sendonaris, E. Erkrip, and B. Aazhang, "User cooperation diversityPart I: System description," IEEE Trans. Commun., vol. 51, pp. $1927-$ 1938, Nov. 2003.

[4] — "User cooperation diversity-Part II: Implementation aspects and performance analysis," IEEE Trans. Commun., vol. 51, pp. 1927-1938, Nov. 2003.

[5] J. N. Laneman and G. W. Wornell, "Energy-efficient antenna sharing and relaying for wireless networks," in Proc. IEEE Wireless Communications and Networking Conf. (WCNC'00), Chicago, IL, Oct. 2000, pp. 7-12.

[6] J. N. Laneman, D. N. C. Tse, and G. W. Wornell, "Cooperative diversity in wireless networks efficient protocols and outage behaviour," IEEE Trans. Inform. Theory, vol. 50, pp. 3062-3080, Dec. 2004.

[7] R. U. Nabar, H. Bölcskei, and F. W. Kneubühler, "Fading relays channels: Performance limits and space-time signal design," IEEE J. Select. Areas Commun., vol. 22, pp. 1099-1109, Aug. 2004.

[8] M. O. Hasna and M.-S. Alouini, "End-to-end performance of transmission systems with relays over Rayleigh fading channels," IEEE Trans. Wireless Commun., vol. 2, pp. 1126-1131, Nov. 2003.

[9] — "Harmonic mean and end-to-end performance of transmission systems with relays," IEEE Trans. Commun., vol. 52, pp. 130-135, Jan. 2004.

[10] - "Outage probability of multihop transmission over Nakagami fading channels," IEEE Commun. Lett., vol. 7, pp. 216-218, May 2003.

[11] I. S. Gradshteyn and I. M. Ryzhik, Table of Integrals, Series, and Products, 6th ed. New York: Academic, 2000.

[12] M. O. Hasna and M.-S. Alouini, "A performance study of dual-hop transmissions with fixed gain relays," IEEE Trans. Wireless Commun., vol. 3, pp. 1963-1968, Nov. 2004.

[13] M. K. Simon and M.-S. Alouini, Digital Communication over Fading Channels, 2nd ed. New York: Wiley, 2005. 